ORIGINAL ARTICLE

\title{
Increased pulmonary arterial pressure in children with nephrotic syndrome
}

\author{
Z-D Du, L Cao, L Liang, D Chen, Z-Z Li
}

See end of article for authors' affiliations

.....................

Correspondence to: Professor Z-D Du,

Professor of Pediatrics,

Vice Chairman, Children's

Heart Center, Beiijing

Children's Hospital, No.

56, South Lishi Road,

Western District, 100045

Beiijing, China; duzd@

imicams.ac.cn

Accepted 19 January 2004

\begin{abstract}
Aims: To evaluate the pulmonary arterial pressure in children with nephrotic syndrome (NS).
Methods: Doppler echocardiography was performed in 40 children with NS (aged 1.5-13 years) at NS onset $(n=28)$ or relapse $(n=12)$, and 40 normal controls. Pulmonary pressure was estimated by: (1) measuring the systolic transtricuspid gradient from tricuspid regurgitation; and (2) measuring the time to peak velocity of pulmonary flow.

Results: Thirty five of the 40 patients with NS had measurable tricuspid regurgitation with a pulmonary systolic pressure ranging from 21 to $48 \mathrm{~mm} \mathrm{Hg}$. Pulmonary systolic pressure was $>40 \mathrm{~mm} \mathrm{Hg}$ in seven patients. The pulmonary time to peak velocity was shortened and the ratio of time to peak velocity and right ventricular ejection time decreased compared with controls. The patients with increased pulmonary pressure had a longer time since onset of NS. One patient developed thrombus in the inferior vena cava during hospitalisation.

Conclusion: Pulmonary arterial pressure was increased in children with NS. Further work is needed to evaluate the aetiology and clinical implications of this abnormality.
\end{abstract}

$\mathrm{N}$ ephrotic syndrome (NS) is associated with hypercoagulability and an increased tendency for thromboembolism. ${ }^{1-4}$ The reported incidence of thromboembolic complications ranged from $1.8 \%$ to $6.6 \%$ in children with NS. ${ }^{14}$ Although thromboembolism may occur anywhere, deep vein thrombosis and pulmonary embolism are most frequently encountered in the clinical setting. ${ }^{4-6}$ Severe pulmonary embolism is a critical condition which needs emergency intervention, but minor embolism to the pulmonary arteries may not induce clinical symptoms, and occurs in up to $28 \%$ of patients by radionuclide lung perfusion study. ${ }^{6}$ Recurrent pulmonary embolism as well as systemic hypertension may induce increased pulmonary pressure. ${ }^{7}$ However, no systemic studies of pulmonary pressure have been reported in the literature.

By measuring tricuspid regurgitation and Doppler flow of the pulmonary artery, echocardiography has proved to be an accurate and reliable method for assessment of pulmonary artery pressure. ${ }^{8-10}$ We therefore conducted this study in order to evaluate pulmonary artery pressure with Doppler echocardiography in children with NS, and to analyse the influencing factors.

\section{METHODS \\ Subjects}

Forty consecutive children ( 32 males, 8 females) with NS at onset or relapse, admitted to our nephrology unit, were prospectively studied from August 1998 to August 1999. The age range was $1.5-13$ years (mean 7.3 (SD 3.1) years). NS was defined by heavy proteinuria (urine protein $>1 \mathrm{~g} / \mathrm{m}^{2}$ per day), hypoalbuminaemia (serum albumin $<2.5 \mathrm{~g} / \mathrm{dl}$ ), hypercholesterolaemia, and oedema. ${ }^{11}$ The initial treatment comprised $2 \mathrm{mg} / \mathrm{kg} /$ day (maximum $60 \mathrm{mg} /$ day) of prednisone given in three divided doses orally for four weeks, followed by alternate day therapy for another four weeks; daily dose was then tapered for 4-6 weeks and stopped. Response was defined as disappearance of proteinuria $\left(<4 \mathrm{mg} / \mathrm{m}^{2}\right.$ per day on three consecutive days). The patients who still had proteinuria at the end of four weeks of prednisone treatment were considered steroid resistant. In steroid resistant patients and those with frequent relapses, intravenous pulse methylprednisolone or cyclophosphamide was used for the treatment. ${ }^{12}$

Forty age and gender matched normal subjects from a local school or kindergarten served as controls. These subjects had normal blood pressure; no clinical, electrocardiographic (ECG), or echocardiographic evidence of cardiovascular disease; and no evidence of pulmonary disease. Informed consent was obtained from the patients and controls or their guardians. The study had the approval of the Hospital Human Investigation Review Board.

\section{Echocardiography}

Echocardiography was performed on a Toshiba SSH-140 colour Doppler ultrasound system with transducers of $3.75 \mathrm{MHz}$ or $5 \mathrm{MHz}$ as appropriate for children or adolescents. A complete echocardiographic examination was performed to exclude the possibility of congenital heart disease; it was recorded on videotape with a speed of $100 \mathrm{~mm} / \mathrm{sec}$ for Doppler spectrum. The measurement was later done with the incorporated analysis package of the machine. The maximal velocity of tricuspid regurgitation and means of at least three measurements of the other indexes were accepted as the results.

Left ventricular end diastolic and left atrial cavity dimensions were measured..$^{13}$ A modified Simpson's rule was used to measure the left ventricular ejection fraction. Doppler flow of the pulmonary artery was recorded with pulsed wave Doppler to assess pulmonary arterial pressure. Peak velocity, time to peak velocity, and right ventricular ejection time were measured according to Kitabatake and colleagues. ${ }^{9}$ The ratio of time to peak velocity and right ventricular ejection time was calculated.

In each subject tricuspid regurgitation was carefully detected by colour flow mapping and continuous wave Doppler in parasternal right ventricular inflow tract, apical four chamber view, and short axis view at the aortic valve level. Sometimes an early systolic regurgitant flow was obtained. The maximal velocity could not be measured in this situation. Modulation of the transducer or sampling line 
usually permits recording of a holosystolic regurgitant flow. In three patients intravenous injection of agitated saline into the antecubital vein as a contrast was used to obtain measurable tricuspid regurgitation. ${ }^{14}$ If a holosystolic flow was still not available, regurgitation was thought to be not measurable. By using the modified Bernoulli equation $\left(\Delta \mathrm{P}=4 \mathrm{~V}^{2}\right)$, the maximal transtricuspid gradient was estimated. Pulmonary systolic pressure was computed as the sum of the transtricuspid gradient and right atrial pressure. ${ }^{8}$

Right atrial pressure was estimated by the response of the inferior vena cava to deep inspiration. In children under 5 years of age, inferior vena cave respiratory collapse was recorded by making them smile or scream. When the diameter of the inferior vena cava decreased by $<50 \%$ after deep inspiration, right atrial pressure was defined as 10 $\mathrm{mm} \mathrm{Hg}$; when the diameter decreased by $\geqslant 50 \%$, right atrial pressure was defined as $5 \mathrm{~mm} \mathrm{Hg} .{ }^{15}{ }^{16}$ Increased pulmonary pressure was defined as a pulmonary systolic pressure of 40 mm Hg or greater. ${ }^{19}$

Echocardiography was repeated two weeks after the initial study to further evaluate whether the increased pulmonary pressure was related to oedema or whether other factors occurred at the onset of occurrence of disease. All studies were performed by one observer (ZD) who had no knowledge of the patients' clinical data. The procedure was performed before any anticoagulation treatment, with children in a quiet state. None of them received sedatives for the procedure.

Intraobserver variabilities in measurements of cardiac dimension and Doppler velocities were determined by measuring 10 consecutive children on two separate occasions. The variation coefficients were between $2.8 \%$ and $4.2 \%$ for $\mathrm{M}$ mode and two dimensional echocardiographic indexes, and between $3.1 \%$ and $5.2 \%$ for Doppler indexes.

\section{Laboratory and electrocardiogram study}

Twelve lead ECG and chest $x$ ray were recorded. Blood samples for coagulation assays, platelet count, prothrombin time, and biochemical indicators of renal disease (proteinuria, serum albumin, complement C3, cholesterol, creatinine level) were studied.

\section{Statistical analysis}

Data are presented as mean (SD). Means between two groups were compared with the paired or unpaired Student's $t$ test as appropriate. Non-parametric data were compared using the Pearson $\chi^{2}$ test or Fisher exact test. The 95\% confidence intervals (CI) were calculated as appropriate. Linear correlation and regression were used to test the correlations between pulmonary systolic pressure and the independent variables. The Mann-Whitney $U$ test was used to compare data that were not normally distributed. SPSS 10.0 for Windows (SPSS Inc., Chicago) was used for the analysis. A p value of 0.05 was regarded as significant.

\section{RESULTS}

\section{Clinical features}

Of the 40 patients, 39 had no identified aetiology and were considered as having idiopathic NS; one had a positive hepatitis B virus surface antibody. Twenty eight patients were at NS onset; 12 were patients during relapses after remission. The time from onset of the disease ranged from 1 day to 11 years with a median of 3 weeks. At the time of admission, all patients had oedema, heavy proteinuria $\left(2.5(1.6) \mathrm{g} / \mathrm{m}^{2}\right.$ per 24 hours), hypoalbuminaemia (1.9 (0.6) g/dl), and hypercholesterolaemia (448 (176) $\mathrm{mg} / \mathrm{dl})$. Serum creatinine ranged from 0.35 to $1.30 \mathrm{mg} / \mathrm{dl}(0.54(0.25) \mathrm{mg} / \mathrm{dl})$. Total platelet count and prothrombin time were $32.6(9.9) \times 10^{4} / \mathrm{mm}^{3}$ and 13.3 (1.4) seconds, respectively. Mean fibrinogen level ranged from 0.08 to $0.75 \mathrm{~g} / \mathrm{dl}(0.36(0.13) \mathrm{g} / \mathrm{dl})$. Chest $x$ ray showed 29 patients had pulmonary congestion.

A total of 30 patients had a response during initial treatment; 10 were steroid resistant. In patients with steroid resistance, data from a percutaneous kidney biopsy were available in only three patients: in two shown to be membranoproliferative glomerulonephritis; and one had minimal change disease. Intravenous pulse methylprednisolone and cyclophosphamide were used in 14 and 7 patients, respectively. During hospitalisation, one patient developed a thrombus in the inferior vena cava. The patient was a 10 year old boy with minimal change NS at his second relapse. He had severe oedema, and was documented with a thrombus by Doppler ultrasound. After heparin therapy, the thrombus shrank as assessed by ultrasound two weeks later. The patients did well at the two month follow up. None in this group received any medicines for their systemic hypertension.

ECG was abnormal in 12 patients. Of these, five had frequent ventricular or atrial $(\mathrm{n}=1)$ premature beats, and seven had ST or T wave changes. In comparison to normal controls, patients with NS had a larger left atrial and right ventricular dimension by echocardiography, and higher systolic and diastolic blood pressure (table 1).

\section{Pulmonary pressure estimated by tricuspid regurgitation}

Of the 40 patients, 37 had tricuspid regurgitation. The regurgitation was not measurable in two patients. The remaining 35 patients with holosystolic tricuspid regurgitation were used for estimation of pulmonary pressure. The maximal regurgitant velocity was $2.0-3.3 \mathrm{~m} / \mathrm{sec}(2.6(0.3)$ $\mathrm{m} / \mathrm{sec}$ ), with a transtricuspid gradient of $16.0-42.5 \mathrm{~mm} \mathrm{Hg}$ (28.3 (7.2) $\mathrm{mm} \mathrm{Hg}$ ).

Right atrial pressure was assessed as $5 \mathrm{~mm} \mathrm{Hg}$ in 37 and 10 $\mathrm{mm} \mathrm{Hg}$ in 3 patients, respectively. Pulmonary systolic pressure ranged from 21.0 to $47.5 \mathrm{~mm} \mathrm{Hg}$ (33.7 (7.4) $\mathrm{mm} \mathrm{Hg})$. Pulmonary systolic pressure was $>30 \mathrm{~mm} \mathrm{Hg}$ in 13 patients $(32.5 \%, 95 \%$ CI $19.1 \%$ to $41.2 \%$ ), and $>40 \mathrm{~mm} \mathrm{Hg}$ in 7 patients $(17.5 \%, 95 \%$ CI $7.9 \%$ to $33.4 \%)$. The sole patient with inferior vena cava thrombus had a pulmonary systolic pressure of $41 \mathrm{~mm} \mathrm{Hg}$.

Of 40 normal controls, 28 had tricuspid regurgitation, immeasurable in three. All had an estimated right atrial pressure of $5 \mathrm{~mm} \mathrm{Hg}$. Pulmonary systolic pressure ranged from 17.0 to $32.3 \mathrm{~mm} \mathrm{Hg}(24.2$ (4.7) $\mathrm{mm} \mathrm{Hg})$, and was

Table 1 Clinical and echocardiographic data of 40 children with nephrotic syndrome compared with controls

\begin{tabular}{lllr}
\hline & $\begin{array}{l}\text { Patients with } \\
\text { NS }(\mathbf{n}=40)\end{array}$ & $\begin{array}{l}\text { Controls } \\
(\mathbf{n}=40)\end{array}$ & p value \\
\hline Age (years) & $7.1(3.1)$ & $7.1(3.1)$ & 0.738 \\
BSA (m²) & $0.91(0.22)$ & $0.90(0.20)$ & 0.983 \\
Heart rate (beats/min) & $96(18)$ & $92(13)$ & 0.245 \\
SBP $(\mathrm{mm} \mathrm{Hg})$ & $108(13)$ & $93(9)$ & $<0.001$ \\
DBP (mm Hg) & $71(11)$ & $60(8)$ & $<0.001$ \\
Left atrial dimension (mm) & $24.3(3.0)$ & $20.7(2.6)$ & $<0.001$ \\
LVEDD (mm) & $36.9(4.5)$ & $36.9(4.4)$ & 0.988 \\
RVEDD (mm) & $12.0(2.2)$ & $10.9(2.3)$ & 0.038 \\
LVEF (\%) & $70.8(4.8)$ & $68.3(8.0)$ & 0.181 \\
PA flow velocity (m/s) & $1.01(0.14)$ & $1.00(0.14)$ & 0.841 \\
PAT (ms) & $98(20)$ & $114(28)$ & 0.007 \\
RVET (ms) & $290(31)$ & $295(24)$ & 0.409 \\
PAT/RVET & $0.34(0.07)$ & $0.39(0.09)$ & 0.007 \\
\hline
\end{tabular}

Data are presented as mean (SD).

BSA, body surface area; SBP, systolic blood pressure; DBP, diastolic blood pressure; LVEDD, left ventricular end diastolic dimension; RVEDD, right ventricular end diastolic dimension; LVEF, left ventricular ejection fraction; NS, nephrotic syndrome; PA, pulmonary artery; PAT, pulmonary arterial flow acceleration time; RVET, right ventricular ejection time. 


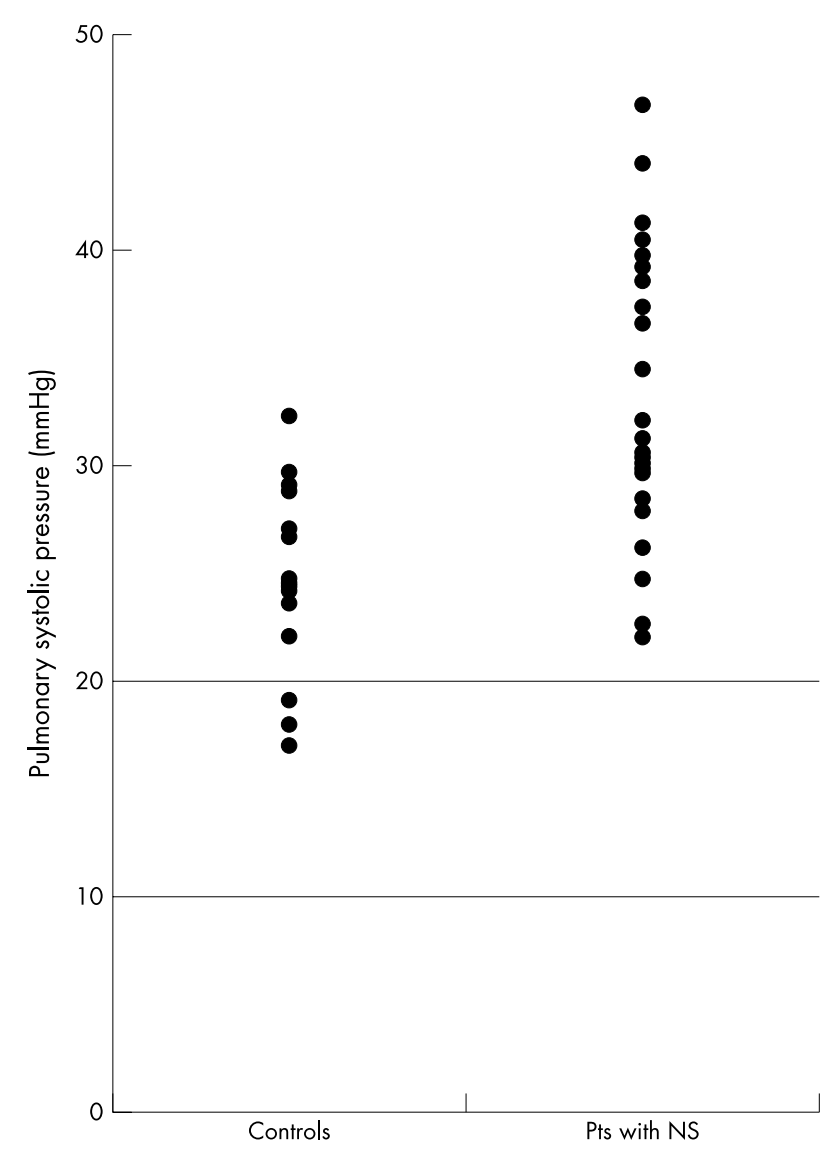

Figure 1 Comparison of pulmonary arterial systolic pressure between children with nephrotic syndrome and normal controls.

significantly lower than that in the patients with NS $(\mathrm{p}<0.01)$ (fig 1). Only one normal child had a estimated pulmonary systolic pressure above $30 \mathrm{~mm} \mathrm{Hg}$ (32.3 $\mathrm{mm} \mathrm{Hg}$ ).

\section{Pulmonary pressure estimated by Doppler flow in the pulmonary artery}

The time to peak velocity of pulmonary blood flow and the ratio of the time to peak velocity and right ventricular ejection time were obtained in all patients with NS and normal controls. Compared to the normal controls, the time to peak velocity was shortened and the ratio of the time to peak velocity and right ventricular ejection time was decreased in patients with NS (table 1).

\section{Risk factors of increased pulmonary pressure}

Assuming that all patients with no tricuspid regurgitation or with immeasurable regurgitation had normal pulmonary pressure, comparisons of clinical features between patients with increased pulmonary pressure and those with normal pulmonary pressure are shown in table 2. Patients with increased pulmonary pressure had a longer time since their onset of disease $(p=0.003)$. No differences were found in age, whether the patients were at their NS onset or relapse, all biochemical indicators of NS and coagulation, and whether the patients had pulmonary congestion (all $\mathrm{p}>0.05$ ).

Further comparisons between the two groups with respect to cardiac signs and cardiac laboratory test results showed there were no significant differences in left ventricular systolic and diastolic function, blood pressure, and all other parameters. Pulmonary systolic pressure only correlated weakly with right ventricular dimension $(r=0.38$,
Table 2 Comparison of clinical features between patients with increased pulmonary systolic pressure and those with normal pulmonary pressure

\begin{tabular}{|c|c|c|c|}
\hline & $\begin{array}{l}\text { Patients with } \\
\mathrm{PH}(\mathrm{n}=7)\end{array}$ & $\begin{array}{l}\text { Patients without } \\
\text { PH }(n=33)\end{array}$ & p value \\
\hline Age (years) & $6.9(3.1)$ & $7.2(3.1)$ & 0.813 \\
\hline Steroid resistant & $3(42.8)$ & $7(21.2 \%)$ & 0.128 \\
\hline Relapse & $4(57.1 \%)$ & $8(24.2 \%)$ & 0.102 \\
\hline Duration (weeks)* & $13.6(2.0,528.0)$ & $1.5(0.2,92.4)$ & 0.003 \\
\hline Proteinuria $\left(\mathrm{g} / \mathrm{m}^{2} / 24 \mathrm{~h}\right)$ & $2.45(2.11)$ & $2.64(1.54)$ & 0.812 \\
\hline Haemoglobin (g/dl) & $12.7(2.2)$ & $13.3(1.8)$ & 0.474 \\
\hline Platelet count $\left(10^{3} / \mu \mathrm{l}\right)$ & $297(104)$ & $338(102)$ & 0.384 \\
\hline Serum fibrinogen $(\mathrm{g} / \mathrm{dl})$ & $0.36(0.12)$ & $0.39(0.17)$ & 0.721 \\
\hline Cholesterol (mg/dl) & $402.9(155.4)$ & $456.4(159.3)$ & 0.460 \\
\hline Serum albumin $(\mathrm{g} / \mathrm{dl})$ & $1.9(0.7)$ & $1.9(0.6)$ & 0.876 \\
\hline Serum creatinine (mg/dl) & $0.43(0.04)$ & $0.56(0.27)$ & 0.490 \\
\hline $\begin{array}{l}\text { Complement C3 level } \\
(\mathrm{mg} / \mathrm{dl})\end{array}$ & $117(24)$ & $119(27)$ & 0.321 \\
\hline Prothrombin time (s) & $14.4(2.2)$ & $13.5(1.2)$ & 0.181 \\
\hline Pulmonary congestion & $5(71.4 \%)$ & $24(72.7 \%)$ & 0.941 \\
\hline
\end{tabular}

Data are presented as mean (SD).

*Duration (the time from onset of the disease) was presented as median (5th, 95th centile), and was compared using the Mann-Whitney $U$ test. BSA, body surface area; PH, pulmonary systolic pressure $>40 \mathrm{~mm} \mathrm{Hg}$.

Table 3 Comparison of cardiac examination results between patients with increased pulmonary pressure and those with normal pulmonary systolic pressure

\begin{tabular}{llll}
\hline & $\begin{array}{l}\text { Patients with } \\
\text { PH }(\mathbf{n}=7)\end{array}$ & $\begin{array}{l}\text { Patients without } \\
\text { PH }(\mathbf{n}=33)\end{array}$ & p value \\
\hline Heart rate (beats/min) & $99(23)$ & $98(17)$ & 0.889 \\
SBP (mm Hg) & $104(11)$ & $107(13)$ & 0.634 \\
DBP (mm Hg) & $68(10)$ & $69(12)$ & 0.848 \\
Abnormal ECG changes & $2(28.6 \%)$ & $10(30.3 \%)$ & 0.934 \\
Left atrial dimension (mm) & $25.2(2.7)$ & $24.0(3.1)$ & 0.370 \\
Aortic diameter (mm) & $19.1(2.1)$ & $18.8(3.1)$ & 0.787 \\
Pulmonary artery & $16.2(3.8)$ & $16.1(2.3)$ & 0.912 \\
diameter (mm) & & & \\
LVEDD (mm) & $37.4(5.1)$ & $36.1(4.3)$ & 0.478 \\
RVEDD (mm) & $13.3(3.0)$ & $11.6(2.0)$ & 0.074 \\
LVEF (\%) & $68.4(2.9)$ & $708(5.3)$ & 0.273 \\
ME (m/sec) & $1.14(0.25)$ & $1.08(0.21)$ & 0.615 \\
MA (m/sec) & $0.69(0.14)$ & $0.76(0.20)$ & 0.438 \\
ME/A & $1.77(0.74)$ & $1.51(0.41)$ & 0.239 \\
MEDT (msec) & $158(66)$ & $143(24)$ & 0.122 \\
\hline
\end{tabular}

Data are presented as mean (SD).

DBP, diastolic blood pressure; LVEDD, left ventricular end diastolic dimension; LVEF, left ventricular ejection fraction; MA, mitral peak flow velocity during atrial contraction; $M E$, mitral peak early diastolic flow velocity; ME/A, the ratio of mitral peak early diastolic flow velocity and peak flow velocity during atrial contraction; MEDT, deceleration time of mitral early diastolic wave; $\mathrm{PH}$, pulmonary systolic pressure $>40 \mathrm{~mm} \mathrm{Hg}$; RVEDD, right ventricular end diastolic dimension; SBP, systolic blood pressure.

$p<0.05)$. No correlation was found between pulmonary systolic pressure and any biochemical indicators of NS or coagulation (all $\mathrm{p}>0.05$ ).

\section{Follow up data on pulmonary pressure}

Two week follow up echocardiographic data were available in only 18 patients. Of these, 16 had measurable tricuspid regurgitation both at initial and follow up studies. The pulmonary systolic pressure estimated by tricuspid regurgitation did not change significantly $(34.0(8.1) \mathrm{mm} \mathrm{Hg}$ at initial study $v 32.4$ (8.4) $\mathrm{mm} \mathrm{Hg}$ at follow up, $\mathrm{p}>0.05$ ). Pulmonary systolic pressure was $>40 \mathrm{~mm} \mathrm{Hg}$ in five patients. The time to peak velocity of pulmonary blood flow and the ratio of the time to peak velocity and right ventricular ejection time did not change significantly at follow up (all p >0.05). 
At the two week follow up, pulmonary congestion or oedema resolved in all 29 patients with pulmonary congestion documented by chest $x$ ray at the initial study. Of the five patients with increased pulmonary pressure and pulmonary congestion at the initial study, three still had increased pulmonary systolic pressure $(44 \mathrm{~mm} \mathrm{Hg}, 42 \mathrm{~mm} \mathrm{Hg}, 42$ $\mathrm{mm} \mathrm{Hg}$, and $41 \mathrm{~mm} \mathrm{Hg}$, respectively). One had pulmonary systolic pressure of $33 \mathrm{~mm} \mathrm{Hg}$.

\section{DISCUSSION}

Doppler echocardiography has been proved to be a reliable non-invasive method for assessing pulmonary arterial pressure. $^{8-10}$ Although it is not an accurate direct measurement by itself, it has been suggested as the diagnostic method for early detection of primary pulmonary hypertension..$^{18}{ }^{19}$ This study, for the first time, evaluated the pulmonary pressure in children with NS using Doppler echocardiography. By measuring pulmonary blood flow, we found that both the time to peak velocity and the ratio of time to peak velocity and right ventricular ejection time decreased compared to normal controls. This indicated that pulmonary arterial pressure was increased in patients with NS. By measuring the systolic transtricuspid gradient using tricuspid regurgitation, we further found that pulmonary arterial systolic pressure was $>40 \mathrm{~mm} \mathrm{Hg}$ in $17.5 \%$ of patients with NS. This indicated that $17.5 \%$ of the children with NS had increased pulmonary arterial pressure according to the newly recommended criteria of pulmonary hypertension. ${ }^{19}$

The aetiologies of pulmonary arterial hypertension in children with NS may not be concluded from this study; except for the fact that patients with increased pulmonary pressure had a longer time since the onset of disease, we could not find any other factors that predicted or related to the increased pulmonary arterial pressure in children with NS. However, we speculated that the increased pulmonary pressure in children with NS could be ascribed to the following reasons. The first is the hypercoagulable state that is due to the profound abnormalities of almost all coagulation factors and clotting inhibitors, as well as defects in platelets and the fibrinolytic system. ${ }^{1-3}$ Although pulmonary embolism appears to be rare in children compared to adults, ${ }^{4-6}$ the incidence might be underestimated because of the high number of asymptomatic or subclinical events in children with NS. ${ }^{8}$ Even in adults, as many as $50 \%$ of episodes of acute pulmonary thromboembolism still go undetected because the clinical presentation mimics the characteristics of a number of other common and uncommon disease entities, and the sensitivity and specificity of diagnostic tests for pulmonary embolisation remain either weak or poorly defined. ${ }^{20}$ Therefore, some of our patients might have developed pulmonary embolism either during or before the present episode of NS attack.

Our results were not able to identify any relation between increased pulmonary pressure and the biochemical indicators of thromboembolism we studied. This might be due to the large variances of these indicators or the small number of patients with increased pulmonary pressure. As limitations of this study, we did not perform perfusion lung scan or pulmonary artery angiography in each patient, we did not measure the factor $\mathrm{V}$ Leiden mutation, activated protein $\mathrm{C}$ resistance ratio, protein $\mathrm{C}$, protein $\mathrm{S}$, lupus anticoagulant, and other procoagulable states, either. Thus we could not prove that pulmonary embolism is one of the aetiologies for increased pulmonary pressure in children with NS.

The second possible reason for increased pulmonary pressure in children with NS might be systemic hypertension. Blood pressure was higher in children with NS compared to controls. Sustained systemic hypertension has been shown to be a risk factor of increased pulmonary arterial pressure. ${ }^{21} 22$
However, our results did not reveal a statistical difference in blood pressure between patients with normal pulmonary pressure and those with increased pulmonary pressure. Therefore, the length of systemic hypertension might contribute more to the increased pulmonary pressure than the blood pressure itself because patients with increased pulmonary pressure had a longer time since NS onset and larger left atrium size.

The other possible aetiology for increased pulmonary pressure would be the pulmonary interstitial oedema occurring in the acute phase of the disease. After resolution of oedema with diuretics and other treatment, the pulmonary pressure should decrease. However, the follow up data in our 18 patients showed that the pulmonary systolic pressure estimated by tricuspid regurgitation and the time to peak velocity of pulmonary blood flow, and the ratio of the time to peak velocity and right ventricular ejection time did not change significantly from the initial study. Oedema would resolve in most of the patients after treatment. Therefore it seems that the increased pulmonary pressure is not directly caused by pulmonary interstitial oedema during the acute stage.

Although all our patients with increased pulmonary pressure had no clinical symptoms of pulmonary hypertension, it would be important to follow these patients because symptoms and physical findings of pulmonary hypertension are non-specific and can be subtle. As a limitation, the long term prognosis of the increased pulmonary pressure in patients with NS is still unknown from this study. If the increased pulmonary pressure persisted, it could damage the right ventricle and pulmonary functions and may also impair left ventricular filling and ejection fraction. Therefore we suggest that antiplatelet and/or anticoagulation drugs might help in patients with documented hypercoagulability in preventing development of pulmonary hypertension.

Of all our patients, only one presented with deep venous thrombus. The incidence is similar to previous reports. ${ }^{4-6}$ As we did not investigate subclinical episodes of thromboembolism, we could not exclude the possibility that there might be more patients with silent thromboembolitic events.

In conclusion, although we could not clarify the aetiology, this study showed that pulmonary arterial pressure was increased in the $17.5 \%$ of children with NS. Further studies are needed to further evaluate the aetiology, clinical implications, and long term prognosis of this abnormality.

\section{Authors' affiliations}

Z-D Du, Z-Z Li, Children's Heart Center, Beijing Children's Hospital, Capital University of Medical Sciences, Beijing, China

L Cao, L Liang, D Chen, Department of Nephrology, Capital Institute of Pediatrics

\section{REFERENCES}

1 Sagripanti A, Barsotti G. Hypercoagulability, intraglomerular coagulation, and thromboembolism in nephrotic syndrome. Nephron 1995;70:271-81.

2 Cameron JS. Thromboembolic complications of the nephrotic syndrome. Adv Nephrol 1984;13:75-114.

3 Citak A, Emre S, Sairin A, et al. Hemostatic problems and thromboembolic complications in nephrotic children. Pediatr Nephrol 2000;14:138-42.

4 Lilova MI, Velkovski IG, Topalov IB. Thromboembolic complications in children with nephrotic syndrome in Bulgaria (1974-1996). Pediatr Nephrol 2000; 15:74-8.

5 Peces R, Pobes A, Rodriguez M, et al. Multiple venous thrombosis and massive pulmonary artery thrombus as the presenting features of steroid-responsive nephrotic syndrome. Nephrol Dialysis Transplant 1999;14:1306-9.

6 Hoyer PF, Gonda S, Barthels M, et al. Thromboembolic complications in children with nephrotic syndrome. Acta Paediatr Scand 1986;75:804-10.

7 Share A, Uzun O, Blackburn MEC, et al. Prolonged local infusion of streptokinase in pulmonary artery thrombosis with nephrotic syndrome. Pediatr Nephrol 1996; 10:734-6.

8 Yock PG, Popp RL. Noninvasive estimation of right ventricular systolic pressure by Doppler ultrasound in patients with tricuspid regurgitation. Circulation 1984;70:657-62. 
9 Kitabatake $A$, Inoue $M$, Asao $M$, et al. Non-invasive evaluation of pulmonary hypertension by a pulsed Doppler technique. Circulation 1983;68:302-9.

10 Kosturakis D, Goldberg S, Allen H, et al. Doppler echocardiographic prediction of pulmonary artery hypertension in congenital heart disease. Am J Cardiol 1984;53:1110-15.

11 International Study of Kidney Disease in Children. Nephrotic syndrome in children: prediction of histopathology from clinical and laboratory characteristics at the time of diagnosis. Kidney Int 1978;13:159-65.

12 British Association for Paediatric Nephrology and Royal College of Physicians. Consensus statement on management and audit potential for steroid responsive nephrotic syndrome. Report of a Workshop by the British Association for Paediatric Nephrology and Research Unit, Royal College of Physicians. Arch Dis Child 1994;70:151-7.

13 Sahn DJ, Demaria A, Kisslo J, et al. The committee on M-mode standard of the American Society of Echocardiography: recommendations regarding quantitation in M-mode echocardiography: results of a survey of echocardiographic measurements. Circulation 1978;58:1072-83.

14 Beard JT II, Byrd BF II. Saline contrast enhancement of trivial Doppler tricuspid regurgitation signals for estimating pulmonary artery pressure. Am J Cardiol 1988;62:486-8.
15 Simonson JS, Schiller NB. Sonospirometry: a non-invasive method for estimation of mean right atrial pressure based on two-dimensional echographic measurements of the inferior vena cava during measured inspiration. J Am Coll Cardiol 1988;11:557-64.

16 Du ZD, Roguin N, Milgram E, et al. Pulmonary hypertension in patients with thalassemia major. Am Heart J 1997;134:532-7.

17 Rich S. Pulmonary hypertension. In: Braunward E, eds. Heart disease: a textbook of cardiovascular medicine, 6th edn. St Louis: W B Saunders Company, 2001:1908-31.

18 Galie N, Torbicki A. Pulmonary arterial hypertension: new ideas and perspectives. Heart 2001;85:475-80.

19 Rich S. Primary pulmonary hypertension: executive summary from the world symposium - primary pulmonary hypertension 1998. World Health Organisation. http://www.who.int/ncd/cvd/pph.html.

20 Peterson KL. Acute pulmonary thromboembolism: has its evolution been redefined? Circulation 1999;99:1280-3.

21 Littler WA, Redman CW, Ronnar J, et al. Reduced pulmonary arterial compliance in hypertensive pregnancy. Lancet 1973;1:1274-8.

22 Alpert MA, Baver JH, Parker BM, et al. Pulmonary hemodynamics in systemic hypertension. Long-term effect of minoxidil. Chest 1979;76:379-83.

\section{ARCHIVIST}

\section{Outcomes after head injury}

ong term outcome after head injury in childhood is variable and children with even mild

head injuries may have persisting cognitive and behavioural problems. Follow-up studies

Lhave suffered from the absence of widely accepted outcome measures. The Glasgow Outcome Scale provided such a measure for adults and the King's Outcome Scale for Childhood Head Injury (KOSCHI, described in Archives in 2001) has been proposed as a standardised outcome measure for children. The KOSCHI has been used in a study of headinjured children in Stoke-on-Trent (CA Hawley and colleagues. Journal of Neurology, Neurosurgery, and Psychiatry 2004;75:737-42).

Between November 1992 and December 1998 a total of 986 children were admitted to hospital in Stoke with head injury and 12 died. A questionnaire was sent in 1998 to parents of 974 surviving children aged 5-15 years at the time of injury and 526 satisfactory responses were received.. Head injuries were classified as mild $(<15$ minutes unconsciousness, Glasgow Coma Scale (GCS) 13-15), moderate (>15 minutes unconsciousness, GCS 9-12), or severe ( $>6$ hours unconsciousness, GCS 3-8). The KOSCHI scoring system has five categories: (1) death, (2) vegetative, (3) severe disability (A or B), (4) moderate disability (A or $\mathrm{B}$ ), and (5) good recovery (A or B). (In subcategories, A implies a less satisfactory outcome than B.) Of the 526 children whose parents returned a completed questionnaire 419 had had mild head injury, 58 moderate, and 49 severe. Mean follow up was 2.2 years. Severe disability (KOSCHI score 3) was reported for four children, all with severe head injury and good recovery (KOSCHI score 5) for 270 (51\%). Good recovery occurred in 57\% after mild head injury, $36 \%$ after moderate, and $22 \%$ after severe head injury. Complete recovery (KOSCHI score 5B) occurred in 97 children $(18 \% ; 21 \%, 10 \% \text {, and } 4 \%)^{*}$. Social deprivation was associated with poorer outcome. The presence or absence of skull fracture did not influence outcome. Eight children (five, one, and two) developed epilepsy.

Few children changed school because of the head injury but special educational needs (SEN) were identified in $40(23,2$, and 15). Parents reported that SEN provision had been made for 26 children. Ninety-eight children (19\%; 16\%, 28\%, and 35\%) had difficulties with schoolwork and 96 (18\%; 17\%, 24\%, and 23\%) had been disciplined for bad behaviour at school. Only 209 teachers (40\%; 33\%, 55\%, and 77\%) knew about the child's head injury. Change of personality was reported for 148 children $(28 \% ; 21 \%, 46 \%$, and $69 \%)$. Headaches, temper outbursts, and mood swings each occurred in some $13-18 \%$ of children after mild head injury becoming more frequent (up to $28 \%-36 \%$ ) after severe head injury. These symptoms were uncommon among 45 control children.

Forty-three per cent of children with mild head injury had moderately severe persisting disability but many are not followed up. The authors of this paper suggest that a system of postal follow up with a questionnaire incorporating the KOSCHI checklist might identify children who need to be reassessed.

${ }^{*}$ All series are: total; mild, moderate, and severe. 